\title{
Meeting measles elimination indicators: surveillance performance in a regional area of Australia
}

\author{
Julie K Kohlhagen, ${ }^{a}$ Peter D Massey and David N Durrheim ${ }^{a}$ \\ Correspondence to Julie K Kohlhagen (e-mail: Julie.Kohlhagen@hnehealth.nsw.gov.au)
}

The World Health Organization (WHO) Western Pacific Region has established specific measles elimination surveillance indicators. There has been concern in Australia that these indicators may be too stringent and that measles elimination can occur without all surveillance prerequisites being met, in particular the minimum fever and rash clinician-suspected measles reporting rate with subsequent laboratory exclusion of measles. A regional public health unit in northern New South Wales, Australia, prompted local general practitioners to report fever and rash presentations that met the measles case definition or that they considered to be clinical measles. These notifications from July 2006 to June 2008 were reviewed to determine whether measles indicators for monitoring progress towards measles elimination could be achieved in Australia. Results confirmed that the surveillance indicators of ">2 reported suspected measles cases per 100000 population," "at least $80 \%$ of suspected cases adequately investigated within 48 hours" and "greater than $80 \%$ of cases had adequate blood samples collected" could be met. Only half the cases had virology that would allow genotyping of measles virus. Special efforts to engage and convince Australian medical doctors about the public health value of reporting clinically suggestive measles cases and collecting confirmatory blood tests resulted in the current WHO Western Pacific Region indicators for progress towards measles elimination being met in a regional area of Australia.

$\mathrm{M}$ easles is a highly infectious viral illness that caused an estimated 164000 deaths worldwide in 2008. ${ }^{1,2}$ As humans are the only natural host of measles virus, there is only a single genetically stable serotype, there is a safe and effective vaccine that provides long-lasting protection, and indigenous measles transmission has been interrupted in the Region of the Americas since 2002, global measles eradication is considered feasible and desirable. ${ }^{3}$ The World Health Organization Western Pacific Regional Committee has established a measles elimination target date of $2012 .^{4}$

Measles continues to occur in Australia with most cases in recent years resulting directly from importation of the virus. In 2009 and 2010, there were 105 and 69 confirmed measles cases notified in Australia, respectively. ${ }^{5}$ Australian researchers claim that measles has been eliminated based on: absence of endemic measles genotype (D1) since 1999; high vaccination coverage (measles-containing vaccine [MCV] first dose coverage $>95 \%$ and MCV2 coverage $>90 \%$ since 2004); serological evidence of $>90 \%$ population immunity and containment of outbreaks without apparent re-establishment of a specific genotype since $1999 .{ }^{6,7}$ The contention is that despite not meeting all Western Pacific Region surveillance targets at the national level there is adequate evidence to justify formal declaration of measles elimination in Australia. ${ }^{7}$

However, during 2011 there has been an increase in measles activity with 82 cases reported to 31 March, with most cases locally acquired without clear epidemiological links apparent between all cases. ${ }^{5}$ An average of 69 measles cases were reported in New South Wales (NSW) each year for the past 10 years. ${ }^{5}$ We reviewed suspected measles cases reported between July 2006 and June 2008 in a regional area of northern New South Wales to determine whether the Western Pacific Region indicators for monitoring progress towards measles elimination were met at the subnational level and what implications there might be for documenting sustained elimination in Australia. During the study period 2006-2008, measles notification rates in Australia were 0.1-0.6 per 100000 population. $^{5}$

\footnotetext{
- Hunter New England Population Health, New South Wales, Australia 


\section{Box 1. Australian measles case definition}

\section{Confirmed case}

A confirmed case requires either:

1. laboratory definitive evidence, OR

2. clinical evidence AND epidemiological evidence.

Laboratory definitive evidence

At least one of the following:

1. Isolation of measles virus, OR

2. Detection of measles virus by nucleic acid testing, OR

3. Detection of measles virus antigen, $\mathrm{OR}$

4. IgG seroconversion or a significant increase in antibody level or a fourfold or greater rise in titre to measles virus EXCEPT if the case has received a measles-containing vaccine 8 days to 8 weeks before testing, OR

5. Detection of measles virus specific IgM antibody confirmed in an approved reference laboratory EXCEPT if the case has received a measles-containing vaccine 8 days to 8 weeks before testing.

Clinical evidence

An illness characterized by all of the following:

1. A generalized maculopapular rash lasting three or more days, AND

2. Fever (at least $38^{\circ} \mathrm{C}$ if measured) at the time of rash onset, AND

3. Cough OR coryza OR conjunctivitis OR Koplik spots.

Epidemiological evidence

An epidemiological link is established when there is:

1. Contact between two people involving a plausible mode of transmission at a time when:

a) one of them is likely to be infectious (approximately 5 days before to 4 days after rash onset), AND

b) the other has an illness that starts within 7 to 18 (usually 10) days after this contact, AND

2. At least one case in the chain of epidemiologically linked cases (which may involve many cases) has laboratory definitive evidence measles.

Probable case

A probable case requires laboratory suggestive evidence AND clinical evidence.

Laboratory suggestive evidence

Detection of measles specific IgM antibody other than by an approved reference laboratory EXCEPT if the case has received a measles-containing vaccine 8 days to 8 weeks before testing.

Clinical evidence

Same as for confirmed case.

\section{Suspected case}

A suspected case requires clinical evidence only.

Clinical evidence

Same as for confirmed case.

\section{METHODS}

Surveillance data on measles is collected in New South Wales under the requirements of the Public Health Act (2010). All patient presentations meeting the measles clinical case definition of the National Notifiable Diseases Surveillance System are required to be reported by doctors, hospital chief executive officers, laboratories, school principals and directors of child care facilities (Box 1). ${ }^{8}$

Hunter New England is located in northern New South Wales and has a total population of 880 000. The Public Health Unit regularly prompts local general practitioners to report fever and rash presentations that meet the measles case definition or that they consider to be clinical measles. All suspected measles notifications to the local Public Health Unit are routinely recorded in a secure dedicated Microsoft Excel 2007 spread sheet, and information on probable and confirmed cases is entered into the NSW Notifiable Conditions Information Management System. Suspected measles cases reported by clinicians were analysed to determine whether they met the clinical case definition for measles notification (Box 1 ). ${ }^{8}$ Additional surveillance data were used to determine the likelihood of measles, including travel out of the area or overseas, exposure to other known measles cases and immunization status, with individuals considered fully immunized if they were age-appropriately immunized with MCV1 and MCV2. ${ }^{9}$ Timing of response, laboratory test requests and results were also reviewed. 
Table 1. Clinical features of measles cases notified by clinicians to the Public Health Unit, Hunter New England Area, 2006 to 2008

\begin{tabular}{lc}
\hline \multicolumn{1}{c}{ Symptoms } & No. of cases \\
\hline $\begin{array}{l}\text { Excluded: no clinical features consistent with } \\
\text { the measles case definition }\end{array}$ & 15 \\
Maculopapular Rash $<3$ days & 30 \\
Maculopapular Rash for $\geq 3$ days & 10 \\
$\begin{array}{l}\text { Maculopapular Rash for } \geq 3 \text { days }+ \text { fever at } \\
\text { rash onset }\end{array}$ & 5 \\
$\begin{array}{l}\text { Maculopapular Rash for } \geq 3 \text { days + fever at } \\
\text { rash onset + cough or coryza or conjunctivitis } \\
\text { or Koplik spots }\end{array}$ & 3 \\
\hline Total & 63 \\
\hline
\end{tabular}

This surveillance project was classified as a quality assurance project by the Hunter New England Health Research Ethics Committee.

\section{RESULTS}

The Public Health Unit received 63 suspected measles notifications during the study period July 2006 to June 2008, and 48 had specimens collected either before notification or after discussion with the Public Health Unit. Notifications were received from general practitioners, pathology laboratories, child care centres, schools and health services.

In addition one young child visiting the area from Europe with his family was reported by a general practitioner after the child presented with fever, maculopapular rash, cough, lethargy, coryza and conjunctivitis. Serology, available within 24 hours of collection, confirmed measles. PCR results were available within seven days and genotype D8 was identified. A total of 161 contacts were identified, with normal human immunoglobulin administered to eight and measles vaccine to 19 . No secondary cases were identified.

\section{Clinical criteria}

Of the 63 reported suspected cases, 15 cases were excluded immediately because they did not have clinical features consistent with the measles definition. Forty-eight cases had clinical or epidemiological evidence suggestive of measles at the time of notification to justify collection of pathology specimens. The main presenting symptom for notification of suspected measles was rash $(100 \%$ of notified cases) (Table 1$)$. On further
Table 2. Age group and immunization status of suspected measles cases with specimens collected, Hunter New England Area, 2006 to 2008

\begin{tabular}{lccccc}
\hline $\begin{array}{c}\text { Age } \\
\text { group }\end{array}$ & $\begin{array}{c}\text { Not } \\
\text { immunized }\end{array}$ & $\begin{array}{c}\text { MMR } x \\
\text { 1 dose }\end{array}$ & $\begin{array}{c}\text { MMR } x \\
\text { 2 doses }\end{array}$ & Unknown & Total \\
\hline$<1$ & 11 & n/a & n/a & 11 \\
$\geq 1-<4$ & 2 & 12 & n/a & 3 & 17 \\
$\geq 4-19$ & 1 & 0 & 11 & & 12 \\
$\geq 20$ & 0 & 0 & 3 & 5 & 8 \\
\hline Total & & & & & 48 \\
\hline
\end{tabular}

investigation some of the rashes were not consistent with a measles rash and not all suspected cases had fever at rash onset. Only three suspected cases fulfilled the National Notifiable Diseases case definition.

\section{Specimens collected for pathology}

Fifty-nine measles diagnostic specimens were collected for the 48 suspected cases and only one case had measles confirmed (measles IgM and PCR both positive). Forty-two of the 48 cases ( $87.5 \%$ ) had serology collected for measles IgM and IgG. Seventeen had urine and nasopharyngeal swabs submitted for PCR. In addition to measles and rubella testing, parvovirus was tested in five suspected measles cases and not detected. Notification of suspected measles cases occurred from one day before specimens were collected to six days after specimens were collected, with the median being less than one day after the specimens were collected. The median time between notifications of suspected cases to receiving a laboratory result was two days.

\section{Age and immunization status}

For the 48 suspected measles cases with specimens collected, 11 were less than 12 months of age and not yet immunized. Thirty-seven cases had immunization status recorded and of these 26 were age-appropriately immunized against measles while three were not age-appropriately immunized (Table 2). Among those not immunized, two were children of conscientious objector parents, and one child was born overseas and not fully immunized according to the Australian schedule.

The project was undertaken during a period where published immunization rates for the study 
Table 3. Hunter New England performance against the elimination indicators proposed by the WHO Western Pacific Region, July 2006 to June 2008

\begin{tabular}{ll}
\hline \multicolumn{1}{c}{$\begin{array}{c}\text { Western Pacific Region indicators } \\
\text { for progress towards measles elimination }\end{array}$} & \multicolumn{1}{c}{$\begin{array}{c}\text { Performance in the Hunter New England } \\
\text { regional area of Australia, June 2006 to July 2008 }\end{array}$} \\
\hline 1. Confirmed measles cases $<1$ per million & $\begin{array}{l}<\text { per million (annualized) } \\
\text { Met }\end{array}$ \\
2. Reported suspected measles cases $>2$ per 100000 & $\begin{array}{l}2.7 \text { per } 100000 \text { population per year (annualized) } \\
\text { Met }\end{array}$ \\
3. At least $80 \%$ of districts reporting $>1$ per 100000 suspected cases & Not applicable \\
4. At least $80 \%$ of suspected cases with adequate investigation within & $100 \%$ \\
48 hours of notification* & Met \\
5. At least $80 \%$ of cases with adequate blood samples collected & $100 \%$ \\
6. At least $80 \%$ of cases with laboratory results within seven days & Met \\
& $\begin{array}{l}100 \% \\
\text { Met }\end{array}$ \\
7. At least $80 \%$ of clusters with samples for virus isolation & No clusters occurred \\
8. Two-dose MCV coverage $>95 \%$ & $91.9 \%$ \\
\hline 9. At least $80 \%$ of clusters with $<10$ cases & Not met \\
10. Absence of endemic measles virus & No clusters occurred \\
\hline
\end{tabular}

* Adequate investigation: collection of essential data elements (date of rash onset, date of specimen collection, vaccination status, date of last vaccination, date of birth or age, sex, district) and search for epidemiologically-linked cases. ${ }^{10}$

area were: $93.6 \%$ of children aged 12 to 15 months (MCV1) and $91.3 \%$ of children aged 72 to 75 months (MCV2). ${ }^{11}$

\section{Western Pacific Region indicators for monitoring progress towards measles elimination}

The area met six of the 10 Western Pacific Region interim measles elimination indicators (Table 3); two were not applicable as no clusters were identified and one indicator was not applicable as the regional area is a single district. Immunization coverage fell short of the $95 \%$ indicator.

\section{DISCUSSION}

Our results confirmed that the surveillance indicators of " $>2$ reported suspected measles cases per 100000 population," "at least $80 \%$ of suspected cases adequately investigated within 48 hours" and "greater than $80 \%$ of cases had adequate blood samples collected" that had not previously been reported at the national level in Australia could indeed be achieved at the subnational level. ${ }^{6}$ As quality surveillance indicators have been met and high immunization coverage has been maintained in this regional area, it is likely that indigenous measles has been eliminated.

Clinicians were often convinced, on the basis of limited clinical features, that a patient had measles. The reliability of clinical diagnosis alone will become progressively insecure as measles becomes increasingly uncommon. A high level of alertness at the primary care level is essential if early detection of imported and secondary cases is to be achieved in an area that has eliminated indigenous measles transmission. ${ }^{12}$ Current clinician awareness in this regional area appears adequate.

The review highlighted the need to gather thorough epidemiological information, risk exposure and immunization history when suspected measles cases are reported. A low threshold for serological or virological testing is required if suspected measles cases are to be excluded as confirmed cases. In Victoria, Australia, specimens negative for measles-specific IgM are routinely tested for rubella and parvovirus B19-specific IgM. ${ }^{13}$ The absence of an endemic measles genotype for at least 12 months has been suggested as an important 
alternative measure of measles elimination. ${ }^{7}$ Only half of the suspected cases had specimens collected for measles genotyping, so applying this endemic measles genotype measure will be difficult if the suspected cases became confirmed cases.

Immunization coverage in Australia has been at $91-92 \%$ since the end of 2003 for the 24 month age group while those in the six year age group remain below 90\%. ${ }^{9}$ Meeting the 95\% Western Pacific Region immunization target will be difficult. It then becomes important to meet the Western Pacific Region quality surveillance indicators for building the evidence that Australia has eliminated measles. ${ }^{14}$

Since the study period, suspected measles notifications have continued at the same rate, and four cases in the study area have been confirmed as measles; all were imported from overseas or other parts of Australia. No secondary cases have occurred and no endemic measles genotype has been isolated.

\section{CONCLUSION}

As Western Pacific Region measles elimination approaches, it is important that all countries achieve the surveillance targets necessary for confirming interruption of indigenous measles transmission, including demonstrating their ability to rapidly investigate and exclude cases meeting the clinical case definition. We found that special efforts to engage and convince Australian medical doctors about the public health value of reporting clinically suggestive measles cases and collecting confirming blood tests resulted in the current Western Pacific Region indicators for progress towards measles elimination being met in a regional area of Australia.

\section{Conflicts of interest}

None declared.

\section{Funding}

None.

\section{References:}

1. Mandell G, Bennett J, Dolin R. Measles. In: Mandell, Doug/as, and Bennett's Principles and Practice of Infectious Diseases, 7th edition. Philadelphia, Churchill Livingstone Elsevier, 2010.

2. Centers for Disease Control and Prevention (CDC). Global measles mortality, 2000-2008. MMWR. Morbidity and Mortality Weekly Report, 2009, 58:1321-1326. pmid:19959985

3. Meeting of the Strategic Advisory Group of Experts on Immunization. November 2010 - summary, conclusions and recommendations. Weekly Epidemiological Record, 2011, 86:10-12.

4. Resolution WPR/RC56.R8. Measles elimination, hepatitis B control, and poliomyelitis eradication. In: Fifty-sixth session of the Regional Committee for the Western Pacific, Noumea, 19-23 September 2005. Report of the regional committee summary records of the plenary meetings. Manila, World Health Organization Regional Office for the Western Pacific, 2005, pp 214-215.

5. National notifiable diseases surveillance system. Canberra, Australian Government Department of Health and Ageing (http://www9.health.gov.au/cda/source/cda-index.cfm, accessed on 15 June 2011).

6. Heywood $A E$ et al. Elimination of endemic measles transmission in Australia. Bulletin of the World Health Organization, 2009, 87:64-71. doi:10.2471/BLT.07.046375 pmid:19197406

7. Kelly $\mathrm{H}$ et al. WHO criteria for measles elimination: a critique with reference to criteria for polio elimination. Euro Surveillance: European Communicable Disease Bulletin, 2009, 14:19445. pmid:20070932

8. Australian national notifiable disease case definitions: Measles case definition. Canberra, Australian Government Department of Health and Ageing (http://www.health.gov.au/internet/main/ publishing.nsf/content/cda-surveil-nndss-casedefs-cd_measl.htm, accessed on 28 June 2011).

9. Australian Government Department of Health and Ageing. Annual report: Immunisation coverage, 2007. Communicable Diseases Intelligence, 2009, 33:170-187. pmid:19877535

10. WHO Regional Office for the Western Pacific. Monitoring measles 4: surveillance and progress towards measles elimination. Measles Bulletin, 2007, 13:1-6 (http://www.wpro.who. int/sites/epi/documents/MeaslesBulletin.htm, accessed on 5 August 2011).

11. Hunter New England Communicable Diseases Bulletin. New South Wales, Hunter New England Population Health, 2007, 167:4 (http://www1.hnehealth.nsw.gov.au/hneph/ CMS/_buy/HNEPH\%20Bulletin\%20-\%20July1.pdf, accessed on 3 August 2011).

12. Durrheim DN, Speare R. Measles elimination-a case definition to enhance surveillance. Communicable Diseases Intelligence, 2000, 24:329-331. pmid:11190813

13. Wang $\mathrm{YH}$ et al. Evaluating measles surveillance using laboratorydiscarded notifications of measles-like illness during elimination. Epidemiology and Infection, 2007, 135:1363-1368. doi:10.1017/S095026880700828X pmid:17352839

14. Field guidelines for measles elimination. Manila, World Health Organization Regional Office for the Western Pacific, 2004 (http:// whqlibdoc.who.int/wpro/2004/929061126X.pdf, accessed on 28 June 2011). 